IOSR Journal of Engineering (IOSRJEN)

e-ISSN: 2250-3021, p-ISSN: 2278-8719

Vol. 3, Issue 9 (September. 2013), ||V1\| PP 44-50

\title{
Load Balancing With Multipath Routing In MANAET
}

\author{
Kunjal, Prof. S. A. Jain \\ Kunjal (student) Prof. S. A. Jain (research scholar) MAE, Alandi (D), University of Pune
}

\begin{abstract}
Mobile ad hoc network is a col lection of wireless mobile nodes, such devices as PDAs, mobile phones, laptops etc. that are connected over a wireless medium. There is no pre-existing communication infrastructure (no access points, no base sta-tions) and the nodes can freely move and self-organize into a network topology. Hence, balancing the load in a MANET is important because the nodes in MANET have limited com- munication resources such as bandwidth, buffer space, and battery power. Most of the current routing protocols for mobile Ad-hoc net- works consider the shortest path which is having minimum hop count as optimal route without considering any partic- ular node's traffic and resulting in degradation of the per-formance by causing serious problems in any perticular mo- bile node like congestion, queuing delay and power depletion. Therefore it is very attractive to investigate Routing protocols which use a Routing Metric to Balance Load in Ad-hoc net-works. This paper discusses about LBMPR (Load balancingwith multipath Routing Protocol) for efficient data transmis- sion in MANETs.
\end{abstract}

Keyterms: - Load Balancing, Mobile Ad hoc Networks, Multipath Routing

\section{INTRODUCTION}

MANET is a ad-hoc wireless network formed by a group of mobile nodes which may not be within the transmis- sion range of each other. MANET is having frequently changing topology. The nodes in MANET are self- configuring, self organizing, self-maintaining and charac- terized by multi-hop wireless connectivity. Mobile nodes in MANET are connected by wireless links and each node act as host end router in the network. MANET is a col- lection of mobile nodes, such as PDAs, mobile phones, laptops which are connected over a wireless medium. The routing protocols in MANET can be categorized in to three different groups: Table Driven/Proactive, On- demand/Reactive and Hybrid routing protocols. In Ta- ble Driven routing protocols, each node stores and main- tains routing information to every other node in the net- work. These are done by periodically exchanging routing table throughout the networks. These protocols main- tain tables at each node which store updated routing information for every node to every another node within the network. In on-demand routing protocols, routes are created when required by the source node, rather than storing up-to-date routing tables. Hybrid routing pro- tocols combine the basic properties of the two classes of protocols. In this paper, we propose a multipath routing protocol, LBMPR, in order to minimize the route break recovery overhead. LBMPR provides most of the inter- mediate nodes on the primary path with multiple paths to destination, along with source node. Primary path is the first path received by source node after initiating the route discovery, which is usually the shortest path. Along with shortetest path it provides multiple path to destination from the source node during the route dis-covery process. All the multiple paths are used for data transmission at a time. Existing protocol SMORT is an extension to the unipath routing protocol AODV. The results are compared with the AODV protocol also because, it is important to know if the multipath protocol provides better scalability than its unipath counterpart. Except that comparision between existing SMORT and LBMPR is considered. This paper is organized as fol- lows. In section 2, we described the related work. Section 3 provides system programmers design, finally we include the comparison of the protocols and conclude the paper.

\section{RELATED WORK}

In this section, we briefly present the research work re- lated to multipath routing in literature Recently, some multipath routing protocols have been proposed for ad hoc networks also. Multipath source routing (MSR) [1,2], extends DSR route discovery and route maintenance phases to compute multiple node-disjoint paths. It also proposes a mechanism to distribute load over multiple paths, based on the RTT measurement. SMR finds max- imally disjoint multiple paths and uses a perpacket allo- cation scheme to distribute data packets on to multiple paths. This enables the effective utilization of network resources and avoids nodes from being congested. SMR computes only two 
paths to each destination. All the above protocols are based on the source routing proto- col DSR. Ad hoc on-demand distance vector multipath (AODVM) [3] is also a multipath routing protocol based on AODV. It proposes a routing framework to provide ro- bustness to route breaks. Many disjoint multipath rout- ing techniques $[5,6,7]$ have been proposed for ad hoc net- works, which have focused on improving the reliability of routing using path disjointness or redundancy. Saha et al.[5] proposed a maximally zone-disjoint multipath routing, which computes a set of zone-disjoint shortest paths for traffic load balancing. The zone-disjointness of paths minimizes the congestion for the traffic sent simultaneously over the multiple paths. Disjoint multipath source routing proposed in [6], statically multiplexes the data traffic over multiple disjoint paths at all nodes on the primary path. It achieves better transport capac- ity by doing so, when compared to the original source routing algorithm, in which packets go on a single path from source to destination. Tsirigos and Haas proposed a disjoint multipath routing protocol that can be used in the presence of frequent topological changes. It uses multiple paths simultaneously, by splitting the informa- tion among the multitude of paths. Disjoint multipath routing [4] proposed by Abbasand Jain tries to reduce the effect of path diminution problem in finding node- disjoint multiple paths. As this routing technique also requires the route request packets to carry the traversed path, it suffers from the same disadvantage as the pre- vious protocol. In [7], Ducatelle et al. propose a hy- brid multipath routing based on ant colony optimization framework for traffic load-balancing. Multipath fresnel zone routing [9] proposed by Liang and Midkiff take the capacity of intermediate nodes into consideration for se-

lecting disjoint multiple paths. It evaluates the capac- ity and the transmitting cost of different intermediate nodes, and formulates endto- end paths of different ca- pacity and cost. Then the protocol forwards the traffic through these different paths, by adjusting the amount of traffic on each path based on path capacity and con-gestion conditions. Papadimitratos et al. [10] proposed a reliable disjoint multipath selection approach using an ef- ficient heuristic mechanism. Roy et al. compared the two disjoint multipath techniques that use omnidirectional and directional antennas, respectively. They showed through simulations that directional antennas help in computing multiple paths efficiently, when compared to omnidirectional antennas. Fault tolerant routing pro- posed by Xue and Nahrstedt [11] uses a path estimation mechanism for selecting a reliable route. $\mathrm{Li}$ and Cuth- bert proposed a stable nodedisjoint multipath routing, which applies the path accumulation feature of DSR to AODV. But, this path accumulation feature requires the route request packet to carry the full path it has tra- versed. This requirement increases the size of route re-quest packet, particularly in large networks where paths between nodes are longer. These large-sized route re- quest packets, which are flooded across the entire net- work for route discovery, increase the routing overhead and thereby limit the network scalability.

\section{PROGRAMMER'S DESIGN}

Proposed protocol system is divided into modules and these modules are integrated together for the execution of the system. The proposed system includes following modules,

\section{Route discovery process}

A node initiates route discovery process, when it wants to communicate to a destination. Route Request Phase Proposed protocol considers heterogeneous systems in network, heterogeneity in terms of transmission power, load battery power etc. It calculates the util- ity of the node based on these factors while route discovery. It selects the most resource rich nodes in the network. Route discovery is performed over a number of different iterations. In the first iter-ation the algorithm allows only the most resource rich, meansthe nodes with the highest required util- ity level; nodes to rebroadcast during the route dis- covery phase. If the first iteration fails to determine a route to the required destination, then the source node reduces the utility level requirement to allow less resource rich nodes to also participate in rout- ing. The source node begins by calculating a utility func- tion and assigns a minimum level of utility to which each node must have in order to be able to rebroad-cast the Route Request (RREQ) message. Differ- ent levels of utility requirement are there to be cho- sen, after which if a route to the required destina- tion is not found, the source node will transmit and RREQ without a utility, i.e. all intermediate nodes are allowed to rebroadcast. Each node forwarding a RREQ stores its location information within the RREQ packet. The receiving node will then check to see if the forwarding nodes location falls within its transmission range. If yes, it updates its route table (i.e. assuming bi-directionality) and rebroad-casts the RREQ packet, or sends back a RREP if a route to the destination is known. Otherwise, it deletes the RREQ. Route request is given an ID. RREQ packet is sent with a field RREQ ID. This field contains the id of route request sent. In below figure, node $\mathrm{S}$ is sending a RREQ to neighbor node $\mathrm{F}$, A and J. First copy of the route request 
sets RREQ ID as ID1. Same as second copy of RREQ sets RREQ ID as ID2. And so on. Number

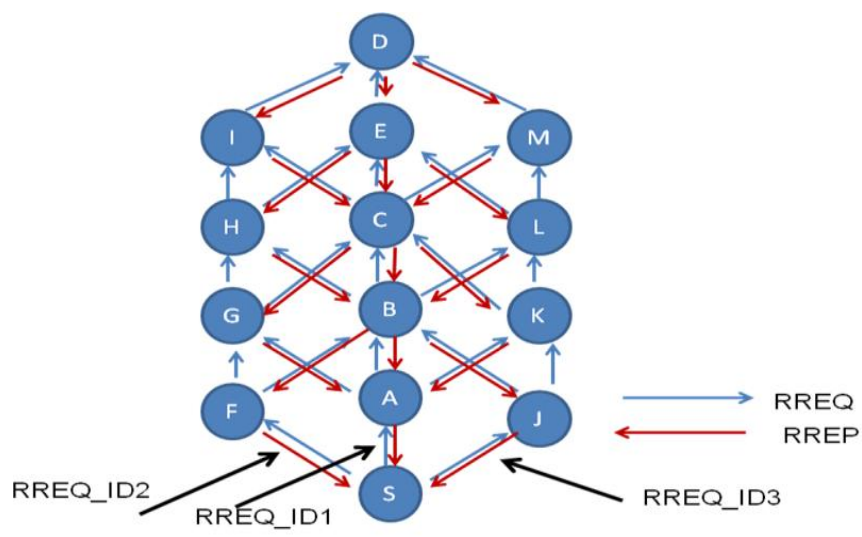

Figure 1: Multiple path between $\mathrm{S}$ and $\mathrm{D}$

of RREQ copies can be restricted. When it comes to route reply the route request which is having ID1 is served first. Then it will reply for ID2.

Route Reply Phase Route replies follow the reverse paths stored in the request-rcvd table to reach the source node. When destination node receives first RREQ packet it will send RREP using reverse path. RREQ ID1 is served first. Consider above figure for the case. Destina- tion node D sends reply for the RREQ ID1. RREP packet consists of mul rply field. For the node $\mathrm{E}$ on the primary path will receive the RREP packet with mul rply value as true. It means it can send multi- ple copies of route reply packet. For the remaining nodes who receive mul rply field as false. It means only one copy of RREP packet can be sent.

Once the path has been discovered destination node will respond to another copy of the RREQ that is RREQ ID2. This time RREQ ID1 will be disabled. Destination node D will send reply for the ID2. The node from which it has received RREQ will receive RREP packet with mul rply value as true. The re- maining nodes will receive the RREP with mul rply value as false. Same procedure will repeat here again. The node with the true value will send multi-

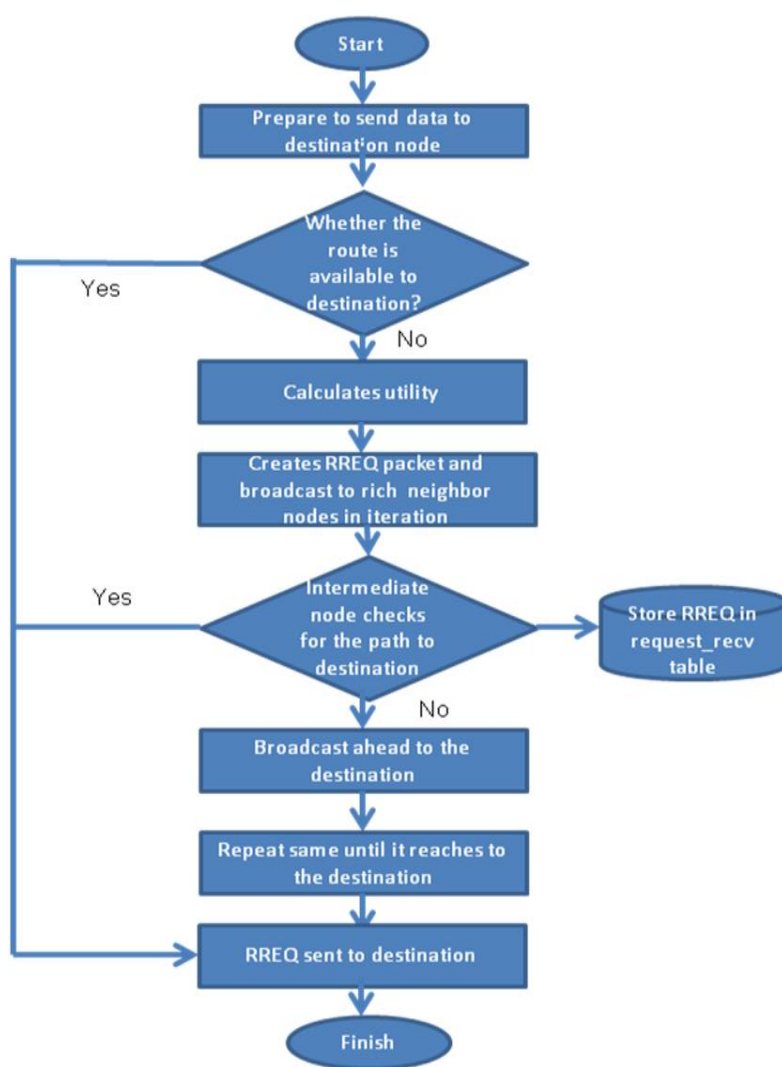

Figure 2: Flowchart-RREQ

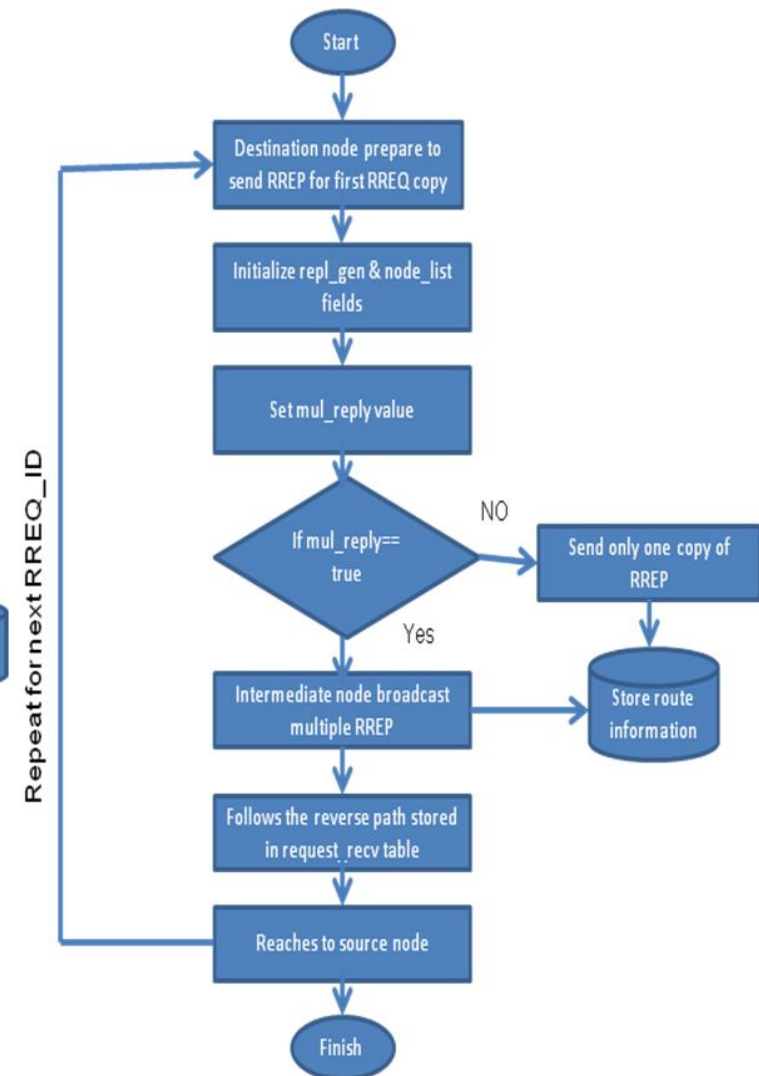

Figure 3: Flowchart-RREP 
ple copy of RREP packet. And the remaining node will send a single copy of RREP packet. Another path will be discovered when this RREP will reach to destination. Same way multiple path can be dis- covered between single source and destination pair.

\section{Data transmission}

Data transmission in proposed mechanism uses traf- fic distribution strategy. The source node uses the technique of traffic splitting to disperse the traf- fic over multiple paths. It is based on weight of the each path. Algorithm uses scheduling technique for time slices. Lets understand with and example. Take a simple example, Path A, B and C, have the weights, 4, 3, 2 respectively, a scheduling sequence will be $\mathrm{AABABCABC}$ in a scheduling period $(\bmod \operatorname{sum}(\mathrm{p}))$. A scheduling sequence will be generated according to the path weights. It calculates the weight by following equation $\mathrm{Temp}_{\mathbf{i}}=\mathrm{BW}_{\mathrm{i}} / \mathrm{L}_{\mathbf{i}} *$ factori sum $=$ Temp $1+$ Temp $2+$ Temp $3+. p_{i}=($ Tempi $/$ sum $) * 100$

Where, BW is the bandwidth and $\mathrm{L}$ is the delay. The path which is having highest weight will be utilize more. The time duration for which the path is used id based upon the $\operatorname{gcd}(\mathrm{p})$.

\section{Route maintenance}

Route maintenance phase maintains the routes es- tablished during the route reply phase, for the time duration of session. The lifetime of routing entries is used for this purpose. The lifetimei of routei rep-resents the time until when the route through nex-thopi is valid. Nodes on the primary path refresh the lifetime of their routing table entries, each time a data packet for the corresponding destination is forwarded. The lifetime of routes at the nodes on the secondary path is initiated to a sufficiently large value. This value can be decided based on the fre- quency of path breaks due to mobility and proba- bility of node failures. We call this parameter as SEC ROUTE LIFETIME. If a requirement for the secondary route arrives before this life time, the sec- ondary route is used for data transmission, and then its lifetime is updated as long as data transmission happens through the route. Otherwise, secondary routes are deleted from routing tables once their ini- tial lifetime expires.

The lifetimei of routei is updated by CURRENT- TIME + ACTIVE-ROUTE-TIMEOUT, whenever a data packet is send through nexthopi successfully. This means that the route is valid and needed till the upcoming ACTIVE-ROUTE-TIMEOUT sec- onds. CURRENT-TIME is the absolute clock time of the nede perferming this update. If a route to the destination expires, that means, if routeâÁźs existing-lifetime is less than CURRENT-TIME, the route is invalid and cannot be used for sending data packets. Later, when a data packet arrives for the same destination, the node checks whether the valid secondary path to the destination is available in the route-list of the routing entry. If a valid secondary route exists, the primary path is replaced with the secondary path and packets are forwarded through it. If a valid secondary does not exist, a route error packet is sent to the source nodes through the nodes in the precur-list of the destinationâĂŹs route entry. And the source node will go for new route discovery.

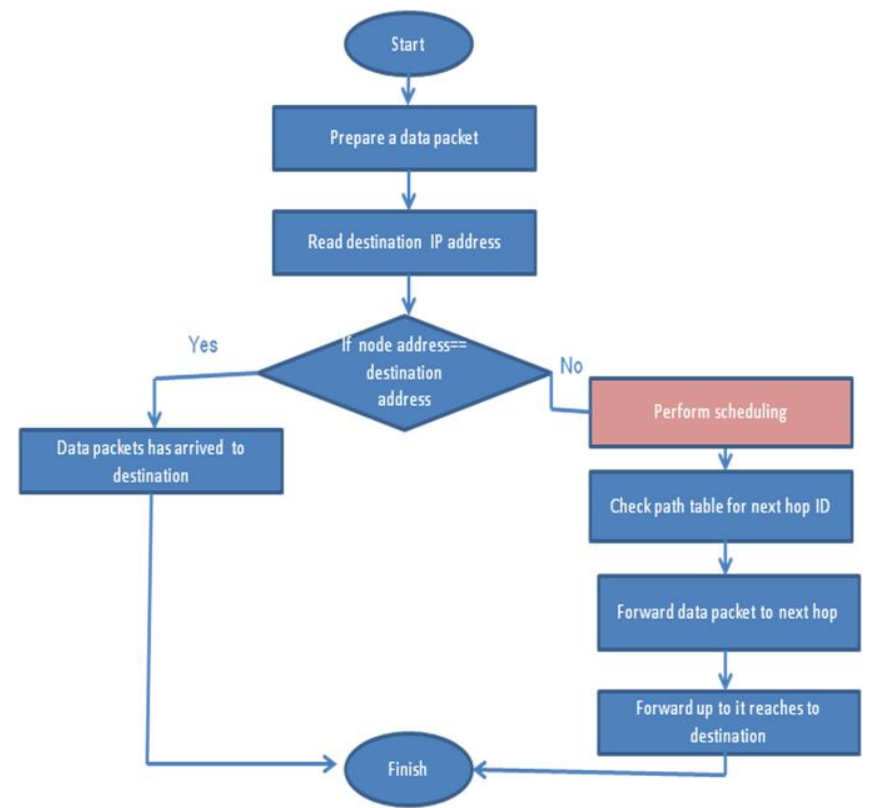

Figure 4: Flowchart-Data transmission 


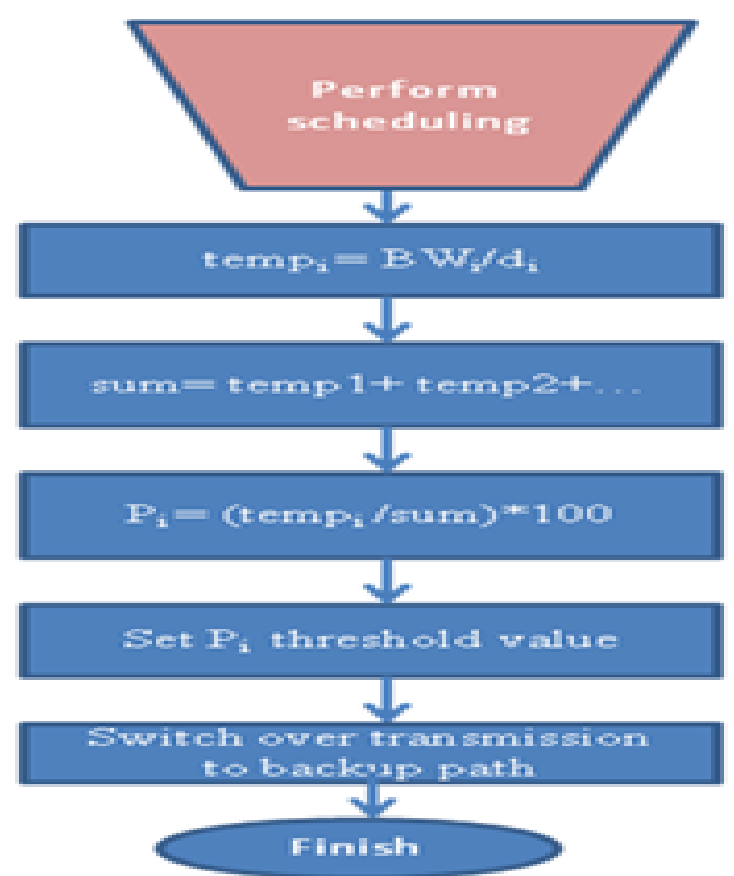

Figure 5: Data transmission

\subsection{Mathematical Model}

Sets for the proposed system Notations

$\mathrm{G}=$ Global set

$\mathrm{N}=$ networks

$\mathrm{WN}=$ Wired Network

WLN= Wireless Network

IN = Infrastructured Network ILN= Insrastructureless Network QOS= Quality Of Service

$\mathrm{D}=$ Delay

$\mathrm{MD}=$ Minimum delay

$\mathrm{MCO}=$ Minimum Control Overhead

$\mathrm{R}=$ Reliability

$\mathrm{LP}=$ Laptop

PDA $=$ Personal Digital Assistant

MOB $=$ Mobile Phones

$\mathrm{D}=$ Delay

RREQ $=$ Route Request Packet RREP $=$ Route Reply Packet DTP = Data Packet

$\mathrm{RERR}=$ Route Error Packet $\mathrm{G}=\{\mathrm{N}\}$

$\mathrm{N}=\{\mathrm{WN}, \mathrm{WLN}\}$ whereN $\subset \mathrm{G}$

$\mathrm{WLN}=\{\mathrm{IN}, \mathrm{ILN}\}$ whereW LN $\subset \mathrm{N}$

$\mathrm{ILN}=\{\mathrm{QOS}, \mathrm{DEV}, \mathrm{PKT}\}$ whereILN $\subset \mathrm{WLN} \mathrm{QOS}=\{\mathrm{D}, \mathrm{MD}, \mathrm{MCO}, \mathrm{R}\} \mathrm{DEV}=\{\mathrm{LP}, \mathrm{PDA}, \mathrm{MOB}\}$

$\mathrm{PKT}=\{\mathrm{RREQ}, \mathrm{RREP}, \mathrm{DTP}, \mathrm{RERR}\}$ whereQOS, DEV, $\mathrm{P}$ K T $\subset$ ILN

\section{Input/output for the proposed system}

For the Route discovery phase Input will be RREQ Packets

Output will be Route between nodes For the Data transmission phase Input will be data Packets sent

Output will be data Packets received For the Route maintenance phase Input will be RERR Packets

Output will be Act to discover alternate path

\section{Functionality}

1. Broadcasting Each node in the sytem can broad-cast RREQ for the new route discovery.

2. Check for the available route This functional- ity checks for the available route between source to desired destination.

3. Find Multiple path This functionality finds the mul- tiple path for transmission of data.

4. Find fail safe path this functionality finds the fail safe path for each primary path which is the combi- nation of node disjoint and link disjoint path. 


\subsection{Dynamic Programming and Serialization}

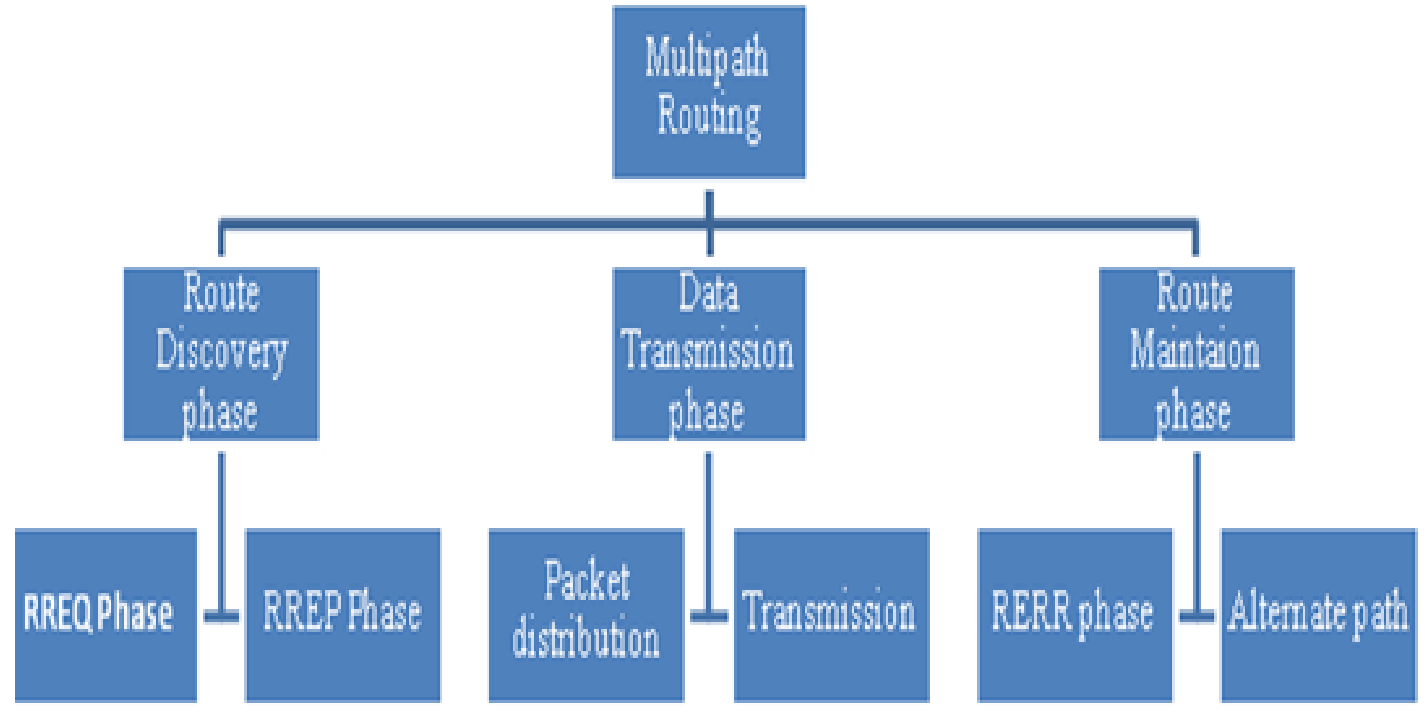

Figure 6: Protocol structure

Here is the structure of protocol which is devided into above mentioned submodules these submodules are merged at the end.

\subsection{Data independence and Data Flow architecture}

The route table is used to store routing information to- wards every destination. Request received table: The table is used to store route request information. Tupple contains (address of the previous node, number of hops). Address of the previous node field represents the node that relayed the route- request to it called lasthop. Number of hops field rep-resents the route-request has traversed from the source node.

Route Table: The route table has an update list of all the possible routes to the desired destinations. Each element in the table is a six tuple of the form (destination addr, route - list, dest seq nb, precurlist). destination addr represent the unique addresses of the destination node; Multiple routes to destination are stored in route - list of the routing entry; The list of lasthops, through which replies are sent, are stored in the precur - list of the rout- ing entry;

\section{Performance metrics}

\section{RESULTS AND DISCUSSION}

Following metrics are going to be compute to eval- uate scalability and performance of SMORT: Throughput : Throughput is calculated as the num- ber of data bytes delivered to all destinations during the simulation.

Average packet transmission delay: Average packet transmission delay is the average time taken by data packets to travel from source node to destination. This per-packet delay includes not only the abso- lute delay experienced by the packet in reaching the destination, but also the delay in resuming the ses- sion, after the route breaks have occurred.

Packet delivery ratio: the ratio of the number of delivered data packet to the destination. This illustrates the level of delivered data to the destination.

2. Experiment Throughput, routing overhead and av- erage packet transmission delay are going to be com- pute by increasing the number of nodes in the net- work from 25,50,75 and 100 nodes. Mobility of nodes will be constant.

\section{Result performance}

Expected result of the above experiment is LBMPR will perform better in terms of above performance matrix.

\section{CONCLUSION}

In this work the objective was to introduce LBMPR. That includes to discover multiple path between source and destination with fail safe path. All the traffic can be transmit through all multiple path at a time. Except this LBMPR considers hetrogeneous nodes in network. 


\section{REFERENCES}

[1] L. Wang, L. Zhang, Y. Shu, M. Dong, Multipath source routing in wireless ad hoc networks, in: Pro- ceedings of Canadian Conference on Electrical and Computer Engineering 2000, vol. 1, March 2000, pp. $479-483$

[2] L. Wang, Y. Shu, M. Dong, L. Zhang, O.W.W. Yang, Adaptive multipath source routing in ad hoc net- works, in: Proceedings of IEEE International Confer- ence on Communications, vol. 3, June 2001, pp. 867-871

[3] Z. Ye, S.V. Krishnamurthy, S.K. Tripathi, A routing framework for providing robustness to node failures in mobile ad hoc networks, Journal of Ad Hoc Net- works 2 (1)

[4] A.M. Abbas, B.N. Jain, Path diminution in disjoint multipath routing for mobile ad hoc networks, in: Proceedings of 15th IEEE International Symposium on Personal, Indoor and Mobile Radio Communica- tions, vol. 1, September 2004, pp. 130 - 134

[5] A. Tsirigos, Z.J. Haas, Multipath routing in mobile ad hoc networks or how to route in the presence of frequent topology changes, in: Proceeding of IEEE Military Communications Conference, vol. 2, Octo- ber 2001, pp. $878-883$

[6] D. Saha, S. Toy, S. Bandyopadhyay, T. Ueda, S. Tanaka, An adaptive framework for multipath rout- ing via maximally zone-disjoint shortest paths in ad hoc wireless networks with directional antenna, in: Proceedings of IEEE Global Telecommunications Conference, vol. 1, December 2003,pp. 226 - 230

[7] N. Wisitpongphan, O.K. Tonguz, Disjoint multipath source routing in ad hoc networks: transport capac- ity, in: Proceedings of IEEE 58th Vehicular Tech- nology Conference, vol. 4, October 2003,pp. $2207-2211$

[8] F. Ducatelle, G.D. Caro, L.M. Gambardella, Ant agents for hybrid multipath routing in mobile ad hoc networks, in: Proceedings of the Second Annual Con-ference on Wireless on demand Network Systems and Services (WONS), January 2005.

[9] Y. Liang, S.F. Midkiff, Multipath fresnel zone routing for wireless ad hoc networks, in: Proceedings of 2005 IEEE Wireless Communications and NetworkingConference, vol. 4, March 2005, pp. 1958âĂŞ1963.

[10] P. Papadimitratos, Z.J. Haas, E.G. Sirer, Path set selection in mobile ad hoc networks, in: MobiHoc 02: Proceedings of the 3rd ACM International Sympo- sium on Mobile Ad hoc Networking and Computing,2004, pp. 23 - 29.

[11] Yuan Xue, K. Nahrstedt, Fault tolerant routing in mobile ad hoc networks, in: 2003 IEEE Wire- less Communications and Networking, vol. 2, March 2003, pp. 1174 - 1179.

[12] L. Reddeppa Reddy *, S.V. Raghavan. 2005 Elsevier 